\title{
Brain stem encephalitis caused by primary herpes simplex 2 infection in a young woman
}

\author{
J W Tang, L J Coward, N W S Davies, A M Geretti, R S Howard, N P Hirsch, K N Ward
}

J Neurol Neurosurg Psychiatry 2003;74:1323-1325

A 27 year old woman developed a vesicular genital rash and cerebellar dysfunction with progressive neurological deterioration suggesting brain stem encephalitis. Respiratory support was required. Magnetic resonance imaging (MRI) of the brain on day 7 showed signal hyperintensity in the central medulla and ventral pons, typical of acute inflammation. The course was severe and relapse occurred. MRI on day 33 showed a haemorrhagic area in the medulla. Treatment with aciclovir/valaciclovir eventually led to gradual recovery. Herpes simplex virus 2 (HSV-2) DNA was detected in CSF on days 11 and 14. HSV-2 was also detected in vesicle fluid from the genital rash. Serum was initially negative for HSV-1 and HSV-2 antibodies, but convalescent samples showed seroconversion to HSV-2, indicating primary infection. Intrathecal synthesis of oligoclonal $\lg G$ bands specific for HSV was identified in the CSF. It is important to differentiate HSV-2 from HSV-1, and primary from initial or reactivated infection, so that prolonged aciclovir treatment followed by prophylaxis is instituted to prevent the high likelihood of symptomatic relapse in primary HSV-2 infection.

$\mathrm{T}$ he incidence of herpes simplex encephalitis is 1 per 250000 to 500000 population per year. ${ }^{1}$ Approximately $33 \%$ of cases occur in patients under the age of 20 years, and about $50 \%$ in those over $50 .{ }^{1}$ Untreated, the mortality for herpes simplex encephalitis is greater than $70 \%$, with only $2.5 \%$ of survivors returning to normality. ${ }^{1}$ Cases occur sporadically and there is no seasonal variation in incidence. Neonatal herpes simplex encephalitis results when HSV is transmitted from the mother to her offspring during childbirth. Herpes simplex virus 2 (HSV-2) accounts for about $80 \%$ of cases in the newborn but after the neonatal period HSV-2 is an uncommon cause of encephalitis, being responsible for less than $10 \%$ of cases. ${ }^{23}$

In adults HSV-2 usually causes uncomplicated genital herpes but occasional cases of neurological involvement are recognised, ranging from meningitis, which may be recurrent, to radiculomyelitis and rarely encephalitis. ${ }^{2-5}$ First infection with HSV-2 as indicated by seroconversion to HSV-2 but in the absence of HSV-l antibody is defined as primary HSV-2 infection and increases the likelihood of disease severity ${ }^{6}$; first infection with HSV-2 as indicated by seroconversion to HSV-2 but in the presence of HSV-1 antibody is defined as initial HSV-2 infection and is usually less severe. ${ }^{6}$ The severity of genital herpes and the likelihood of complications such as meningitis are also greater in women than in men. ${ }^{4}$ Moreover, the more severe an episode of genital herpes the more likely is recurrence in the following weeks and months. ${ }^{4}$

Genital herpes is becoming increasingly common in the United Kingdom, and during 2001 nearly 18000 new diagnoses were made in genitourinary clinics, with most cases seen in young women. ${ }^{7}$ At the same time the proportion of adults lacking HSV-l antibody is increasing, being $46 \%$ of women aged $25-30$ years, ${ }^{8}$ and hence primary HSV-2 infection is becoming more likely.

In this report we describe a case of genital herpes caused by primary HSV-2 infection in a young woman which was complicated by brain stem encephalitis and which required prolonged treatment with aciclovir because of recurrence of herpetic vesicles and relapse of neurological disease.

\section{CASE REPORT}

A 27 year old white woman was referred by her general practitioner to her local hospital with a painful vesicular genital rash. On presentation she was complaining of headache, unsteadiness when walking, double vision, and photophobia. She had no significant previous medical history and, in particular, no history of genital or oral herpes. She was otherwise fit and well and taking no regular drug treatment. She had not travelled outside the United Kingdom in the previous 10 years.

On admission, she was alert and oriented, pyrexial $\left(38^{\circ} \mathrm{C}\right)$, and hypertensive (blood pressure 180/80 mm Hg). An extensive vesicular rash was noted on the posterior aspect of the right thigh, and bilaterally in the sacral and perianal areas. There were no vesicles elsewhere. Neurological examination revealed an intention tremor, marked upper limb and truncal ataxia, oscillopsia, and variable ophthalmoplegia with diplopia and multidirectional nystagmus. There was no evidence of pyramidal weakness or bladder or bowel dysfunction. Other systematic examination was unremarkable. Despite intravenous aciclovir ( $10 \mathrm{mg} / \mathrm{kg}$ three times daily), she went into respiratory failure because of hypoventilation and required tracheal intubation and positive pressure ventilation. In view of this, the patient was transferred to a specialist neurological centre for further management.

On arrival her white cell count was $12.0 \times 10^{9} / 1$ (neutrophils 10.66 and lymphocytes $0.68 \times 10^{9} / \mathrm{l}$, respectively) and $\mathrm{C}$ reactive protein was raised at $72.1 \mathrm{mg} / \mathrm{l}$. Urea, electrolytes, and liver function tests were all within normal limits. An HIV antibody test was negative. Immunoglobulin levels, lymphocyte function, and subsets (CD4 and CD8) were all normal.

Seven days after the onset of the genital rash, magnetic resonance imaging (MRI) of the brain showed signal hyperintensity in the central medulla and ventral pons (fig 1). The appearance was consistent with acute inflammation secondary to infection. Nerve conduction studies were unremarkable and showed no evidence of generalised neuropathy. HSV-2 was cultured from swabs of the patient's vesicular lesions taken on days 7 and 11 . Lumbar puncture was done on days 11 and 14; in each case the cerebrospinal fluid (CSF) showed a predominantly lymphocytic pleocytosis, and HSV-2 DNA was detected by polymerase chain reaction (table 1).

Although continuing to require ventilatory support because of reduced respiratory drive, the patient remained fully alert and her ophthalmoplegia improved. On day 15 of her illness, intravenous aciclovir was stopped because the serum creatinine rose from 69 to $293 \mu \mathrm{mol} / \mathrm{l}$, and urea from 6.5 to 22.6 

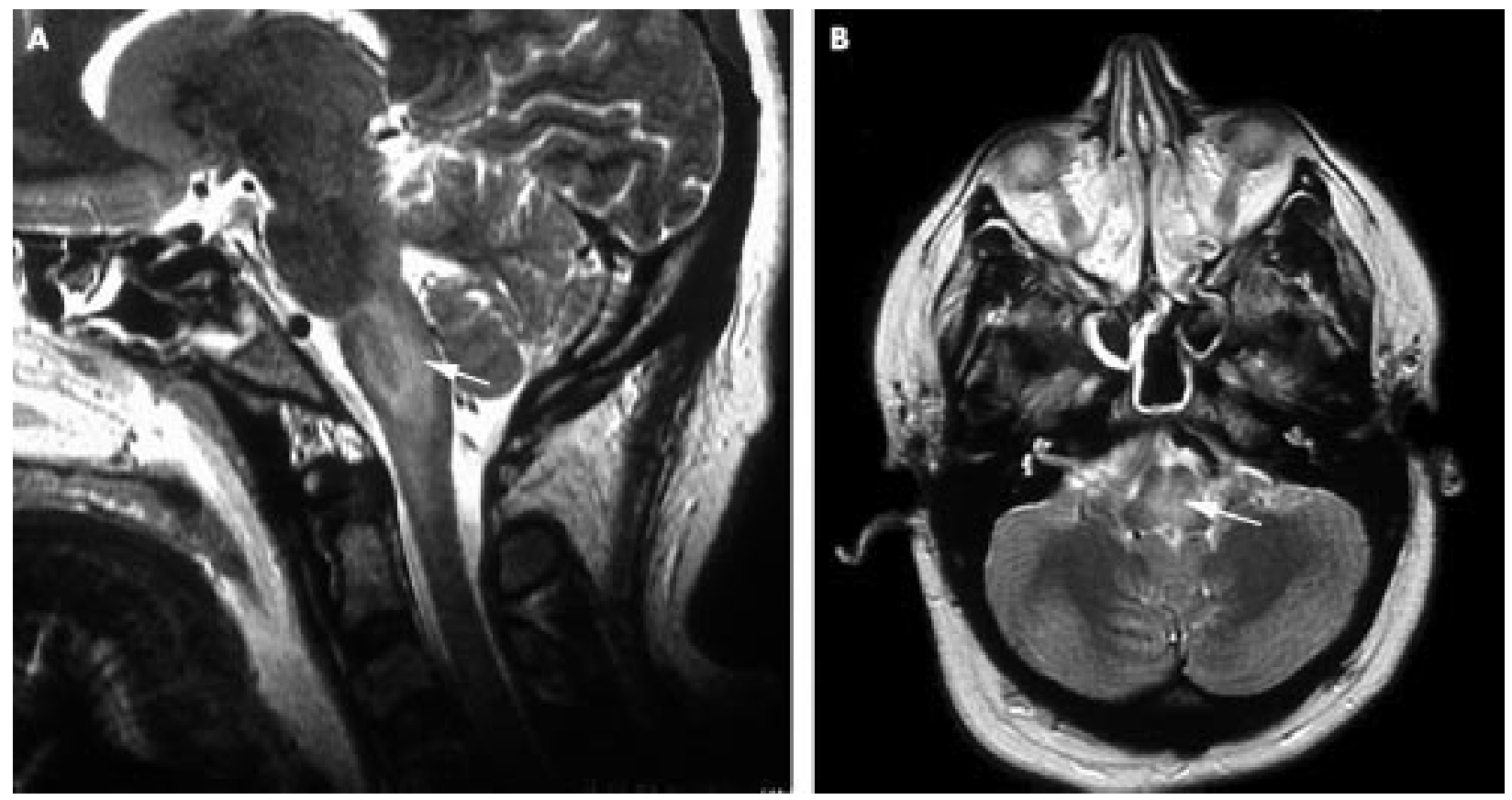

Figure 1 Sagittal (left) and axial (right) magnetic resonance T2 weighted images showing area of high signal (arrow) in the medulla.

$\mathrm{mmol} / \mathrm{l}$. It was restarted on day 20 when renal function had returned to normal. On day 25 it was discontinued (after 15 days of treatment), as a third CSF sample taken on day 20 had been tested for HSV-1 and HSV-2 DNA and found to be negative, although the pleocytosis persisted (table 1).

On day 27, vesicles appeared on the patient's left foot and HSV-2 was cultured from vesicle fluid. Concomitantly, the patient developed worsening upper limb, truncal, and head ataxia, and the new symptom of vertigo. At this point, a fourth CSF sample was taken which still showed a lymphocytic pleocytosis, but was negative for HSV DNA (table 1).

Valaciclovir (500 mg twice daily orally) was started on day 27, as treatment for the relapse of disease. Oral valaciclovir was used as an alternative to intravenous aciclovir because of difficulty with venous access. Repeat brain MRI on day 33 showed haemorrhagic transformation within the original area of hyperintensity in the medulla. From day 60 valaciclovir was discontinued and prophylactic oral aciclovir (400 mg once daily) was given for the next six months. After a period of rehabilitation the patient was finally discharged four months after admission, walking with a rollator frame, with some residual diplopia and dizziness.
The results of laboratory tests on CSF and serum are shown in table 1. Four lumbar punctures were done during the patient's admission and on each occasion her serum was also examined. HSV-2 DNA was found in the first two CSF samples but not in the third or fourth. Oligoclonal IgG bands specific for HSV were detected in the third and fourth CSF samples but were absent from serum. Testing of sequential serum samples showed seroconversion to HSV-2 antibody in the absence of HSV- 1 antibodies, indicating a primary infection.

\section{DISCUSSION}

In this case report we describe a primary HSV-2 infection in an immunocompetent adult which caused a brain stem encephalitis. Primary HSV-2 infection was indicated by seroconversion to HSV-2 antibodies in the absence of antibodies to HSV-1. There was unequivocal evidence of HSV-2 encephalitis as there was both viral invasion of the CNS and an HSV specific intrathecal antibody response. Furthermore, the MRI findings showed a lesion localised to the brain stem which accounted for the patient's neurological signs and symptoms.

Encephalitis limited to the brain stem is a rare presentation of herpes simplex encephalitis-although cranial nerve signs

Table 1 Summary of laboratory tests

\begin{tabular}{|c|c|c|c|c|c|c|c|c|}
\hline \multirow{3}{*}{$\begin{array}{l}\text { Time after } \\
\text { onset of rash } \\
\text { (days) }\end{array}$} & \multicolumn{6}{|l|}{ CSF } & \multicolumn{2}{|l|}{ Serum } \\
\hline & \multicolumn{2}{|c|}{ Cell count (cells/ $\mu$ l) } & \multirow{2}{*}{$\begin{array}{l}\text { Total protein } \\
(\mathrm{g} / \mathrm{l})\end{array}$} & \multirow{2}{*}{$\begin{array}{l}\text { Glucose: } \\
\text { CSF/plasma } \\
\text { (mmol/l) }\end{array}$} & \multirow{2}{*}{$\begin{array}{l}\text { CSF HSV-2 } \\
\text { DNA }\end{array}$} & \multirow{2}{*}{$\begin{array}{l}\text { Oligoclonal IgG bands } \\
\text { unique to CSF and } \\
\text { specific for HSV }\end{array}$} & \multirow[b]{2}{*}{ HSV-1 lgG EIA } & \multirow[b]{2}{*}{ HSV-2 IgG EIA } \\
\hline & White $^{1}$ & Red & & & & & & \\
\hline 6 & & & & & & & $\mathrm{Neg}$ & Neg \\
\hline 11 & 220 & 30 & 2.0 & $4.8 / 8.1$ & $\operatorname{Pos}^{2}$ & No & & \\
\hline 14 & 344 & 1800 & 1.0 & $4.1 / 6.8$ & $\mathrm{Pos}^{2}$ & No & & \\
\hline 20 & 75 & 1000 & 0.25 & $4.0 / 7.1$ & $\mathrm{Neg}^{2}$ & $Y_{e s}^{3}$ & & \\
\hline 27 & 38 & 150 & 0.4 & $3.3 / 5.2$ & $\mathrm{Neg}^{2}$ & $Y_{e s}^{3}$ & & \\
\hline 41 & & & & & & & $\mathrm{Neg}$ & $\operatorname{Pos}^{4}$ \\
\hline 69 & & & & & & & $\mathrm{Neg}$ & $\operatorname{Pos}^{4}$ \\
\hline
\end{tabular}

${ }^{1}$ All white cell counts were predominantly lymphocytes.

${ }^{2}$ Negative for HSV-1, varicella-zoster virus, cyłomegalovirus, and Epstein-Barr virus DNA.

${ }^{3}$ After isoelectric focusing, specificity confirmed by antigen mediated blot to HSV antigen (pooled HSV-1 and HSV-2) as previously described. ${ }^{16}$

${ }^{4}$ Confirmed by immunoblot to HSV-2 antigen.

CSF, cerebrospinal fluid; EIA, enzyme immunoassay; HSV, herpes simplex virus. 
are noted in $32 \%$ of cases of herpes simplex encephalitis, this is more often the result of raised intracranial pressure than of direct viral invasion of the brain stem. ${ }^{9}$ Also, herpes simplex virus brain stem encephalitis confirmed by MRI or necropsy histology usually shows involvement of cerebral cortex. ${ }^{10}{ }^{11}$ Most reports of herpesvirus brain stem encephalitis do not distinguish between HSV-1 and HSV-2. Indeed, there are only two previous reports of HSV-2 brain stem encephalitis, ${ }^{11}{ }^{12}$ and neither of these reports addressed the possibility of primary HSV-2 infection (that is, seroconversion to HSV-2 in the absence of HSV-1 antibodies), as the appropriate serological tests were not done.

The more severe the primary HSV-2 infection the more likely and frequent are recurrent episodes of disease. ${ }^{46}$ In view of this, thorough treatment of encephalitis caused by primary HSV-2 infection is essential. The well established and universally accepted treatment for herpes simplex encephalitis occurring outside the newborn period (normally caused by HSV- 1 ) is intravenous aciclovir ( $10 \mathrm{mg} / \mathrm{kg}$ every eight hours) for 10 to 14 days. ${ }^{13}{ }^{14}$ In our adult case of primary HSV-2 infection, the recommended dose of intravenous aciclovir was given for 10 days followed by a further course of five days. Notwithstanding the apparently successful clearance of HSV from the CSF, the patient developed further vesicles on her left foot from which HSV-2 was cultured, her neurological disease relapsed, and repeat MRI of her brain stem showed haemorrhage in the medulla. Upon immediate treatment with valaciclovir, the vesicles resolved and the neurological symptoms improved markedly. She subsequently made a gradual recovery but was maintained on aciclovir prophylaxis to prevent further episodes of disease.

In this context, it is of interest to consider the latest recommendations for the management of neonatal herpes simplex infections, the majority of which are primary HSV-2 infections. The use of high dose intravenous aciclovir $(20 \mathrm{mg} / \mathrm{kg}$ every eight hours) for 21 days has been shown to reduce morbidity and mortality, and prophylactic aciclovir is under clinical investigation to improve the outcome of those who survive neonatal herpes simplex virus disease. ${ }^{15}$ Thus prolonged high dose intravenous aciclovir followed by oral aciclovir prophylaxis may be justified to prevent relapse and recurrence where primary HSV-2 infection in an adult is accompanied by encephalitis.

\section{Conclusions}

We report a case of HSV-2 brain stem encephalitis in an immunocompetent adult in which the only factors predisposing to severe disease were primary HSV-2 infection and female sex. As far as we are aware this is the first time that brain stem encephalitis has been documented as a result of primary HSV-2 infection. Given the decreasing prevalence of HSV-1 infection in the United Kingdom, primary HSV-2 infection is likely to be an increasing problem in adults. Recognition of neurological disease caused by primary HSV-2 infection is important not only to predict severity, but also as an indication for prolonged aciclovir treatment and prophylaxis.

\section{ACKNOWLEDGEMENTS}

NWSD is funded through a personal training award from the Charitable Foundation of Guy's and St Thomas' Hospital.

\section{Authors' affiliations}

J W Tang, K N Ward, Department of Virology, Royal Free and

University College Medical School, Cleveland Street, London W1, UK

L J Coward, R S Howard, N P Hirsch, Harris Neuromedical Intensive

Care Unit, National Hospital for Neurology and Neurosurgery, Queen

Square, London $\mathrm{WCl}$

N W S Davies, Neurosciences, Guy's, King's, St Thomas' School of

Medicine, Guy's Hospital Campus, London SE 1

A M Geretti, Department of Infection and Public Health Laboratory

King's College Hospital (Dulwich), East Dulwich Grove, London SE22

Competing interests: none declared.

Correspondence to: Dr K N Ward, Department of Virology, Royal Free and University College Medical School, Windeyer Institute of Medical Sciences, 46 Cleveland Street, London W1T 4JF; k.n.ward@ucl.ac.uk

Received 23 January 2003

In revised form 9 April 2003

Accepted 12 April 2003

\section{REFERENCES}

1 Whitley RJ, Gnann JW. Viral encephalitis: familiar infections and emerging pathogens. Lancet 2002;359:507-14

2 Aurelius E, Johansson B, Skoldenberg B, et al. Encephalitis in immunocompetent patients due to herpes simplex virus type 1 or 2 as determined by type-specific polymerase chain reaction and antibody assays of cerebrospinal fluid. J Med Virol 1993;39:179-86.

3 Dennett C, Cleator GM, Klapper PE. HSV-1 and HSV-2 in herpes simplex encephalitis: a study of sixty-four cases in the United Kingdom. J Med Virol 1997:53:1-3.

4 Corey L, Adams HG, Brown ZA, et al. Genital herpes simplex virus infections: clinical manifestations, course, and complications. Ann Intern Med 1983;98:958-72

5 Tedder DG, Ashley R, Tyler KL, et al. Herpes simplex virus infection as a cause of benign recurrent lymphocytic meningitis. Ann Intern Med 1994; 121:334-8

6 Langenberg AGM, Corey L, Ashley R, et al. A prospective study of new infections with herpes simplex virus type 1 and 2. NEng J Med 1999;341:1432-8

7 Public Health Laboratory Service. Genital herpes: epidemiological data. http://www.phls.co.uk/topics_az/hiv_and_sti/sti-herpes/ epidemiology/epidemiology.htm

8 Vyse AJ, Gay NJ, Slomka M, et al. The burden of infection with HSV-1 and HSV-2 in England and Wales: implications for the changing epidemiology of genital herpes. Sex Transm Infect 2000;76:183-7.

9 Whitley RJ, Soong S, Linneman C, et al. Herpes simplex encephalitis. JAMA 1982;247:317-20.

10 Dayan AD, Gooddy W, Harrison MG, et al. Brain stem encephalitis caused by Herpesvirus hominis. BM 1972;iv:405-6.

11 Chu K, Kang D, Lee J, et al. Atypical brainstem encephalitis caused by herpes simplex virus 2. Arch Neurol 2002;59:460-63.

12 Nakajima H, Furutamu D, Shinoda K, et al. Herpes simplex virus type 2 infections presenting as brainstem encephalitis and recurrent myelitis [in Japanese]. Intern Med 1995;34:839-42.

13 Skoldenberg B, Forsgren M, Alestig K, et al. Acyclovir versus vidarabine in herpes simplex encephalitis. Lancet 1984;ii:707-1 1.

14 Whitley RJ, Alford CA, Hirsch MS, et al. Vidarabine versus aciclovir therapy in herpes simplex encephalitis. N Engl J Med 1986;314:144-9. 15 Kimberlin D W. Advances in the treatment of neonatal herpes simplex infections. Rev Med Virol 2001:11:157-63.

16 Monteyne P, Albert F, Weissbrich B, et al, for the European Concerted Action on Virus Meningitis and Encephalitis. The detection of intrathecal synthesis of anti-herpes simplex $\lg G$ antibodies: comparison between an antigen-mediated immunoblotting technique and antibody index calculations. J Med Virol 1997:53:324-31. 